\title{
Using intelligent personal assistants to strengthen the elderlies' social bonds
}

\section{A preliminary evaluation of Amazon Alexa, Google Assistant, Microsoft Cortana, and Apple Siri}

\author{
Arsénio Reis ${ }^{1,2}$, Dennis Paulino ${ }^{1,2}$, Hugo Paredes ${ }^{1,2}$, João Barroso ${ }^{1,2}$ \\ ${ }^{1}$ University of Trás-os-Montes and Alto Douro, Quinta de Prados, 5000-801 Vila Real, Portu- \\ gal \\ ${ }^{2}$ INESC TEC, C Campus da FEUP, Rua Dr. Roberto Frias, 4200 - 465 Porto, Portugal \\ ars@utad.pt, dennis.l.paulinolinesctec.pt, hparedes@utad.pt, \\ jbarrosolutad.pt
}

\begin{abstract}
Social isolation and loneliness are among the important factors for the degradation of the life quality as the persons' aging process advances. These factors can have a pronounced effect on the general health and are caused by the decrease in social interaction by the person with the friends, family and ex-coworkers groups. On the other hand, the software and hardware technologies has reached a maturation point were the electronic assistants can acquire information from the user through camera images, as well as to communicate with the user by means of natural voice language. In this context, a model for the adoption of electronic intelligent assistants by the elderlies has been proposed in previous work. In the current work, it is assessed the possibility of using the current consumer assistants to implement the proposed model. Several assistants are analyzed (Amazon, Google, Microsoft and Apple), assessing their functionalities and how they could be used to assist the elderly in strengthening their social bonds with the family, friends and ex-co-workers groups.
\end{abstract}

Keywords: Wellbeing, Elderly, Ambient Assisted Living, Human Computer Interaction.

\section{Introduction}

Elderly people suffer from an increasing number of problems, mainly due to social isolation and loneliness, requiring support from social agents [1, 2]. These problems, related to loneliness, social isolation, and reduced social activity are linked to the person's mental health, depression, and social bonds [3, 4]. Promoting the social engagement motivates persons to have more complex interactions, mobilizing the cognitive faculties and helping to maintain a good mental health [5]. 
In our previous work [6], it was proposed a model for the design of an autonomous system, based on the paradigm of the intelligent personal assistant, in order to support the elderly people in maintain their social bonds with the family, friends and colleagues groups. This proposal is focused on tailoring the digital assistant for the specific group of elderlies and for their specific life contexts, which has good perspectives, as the intelligent personal assistants are equipment's that are becoming more interactive and with a more natural language [7].

In this work, we assess the possibility of using the intelligent personal assistants, currently available to the consumer public, accordingly to the previously proposed model. The intelligent personal assistants chosen were: Google Assistant [8], Amazon Alexa [9], Apple Siri [10] and Microsoft Cortana [11].These are the most popular and readily available from the large, world class, technology companies.

The Google Assistant is an Intelligent Personal Assistant that allows communication with the user through voice commands. It is capable of search online, set reminders and play music using Spotify. This Intelligent Personal Assistant is integrated with Google Home, Google Allo messaging application and Android Wear (e.g. Smartwatches). The Google Assistant is available in English, German, Hindi, Japanese, and Portuguese. In Fig. 1 it is presented the Google Home Smart Speaker which has builtin Google Assistant.

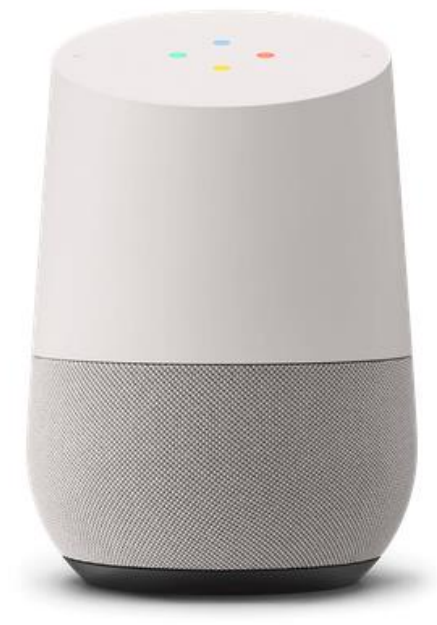

Fig. 1. - Google Home Smart Speaker

The Amazon Alexa is an Intelligent Personal Assistant that interacts with the user with voice commands. It is capable of answer questions online, shop online play and read books. This Intelligent Personal Assistant is integrated with Amazon Echo Smart Speaker, Amazon Fire Devices and Amazon Tap Bluetooth Speakers, it can be installed on Android and iOS. It has also some features useful to integrate with smart homes like 
turning lights on/off or adjusting the temperature at home. The Amazon Alexa is available in English or German. In Fig. 2 it is presented the Amazon Echo Smart Speaker which Amazon Alexa’s integrates.
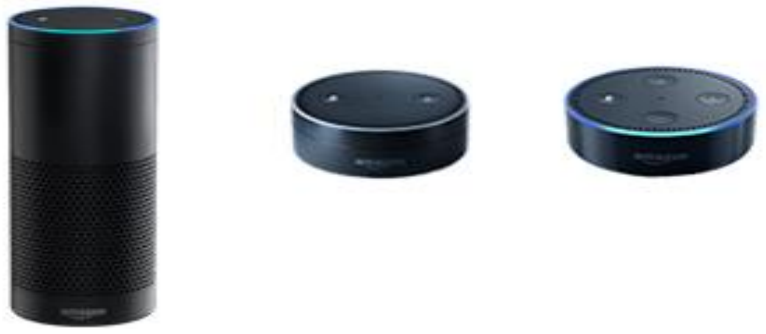

Fig. 2. - Amazon Echo Smart Speaker

The Apple Siri is an Intelligent Personal Assistant that allows communication with the user through voice commands. It is capable of search online, make resevations at restaurants, manage email and make calls. This Intelligent Personal Assistant is integrated with iPhone, iPad and Apple Tv. With the Home app, the user can with Apple Siri control lights,thermostats, door locks and other sensors. The user can communicate with Apple Siri in English, French, Dannish, Finish, Spanish, Japanese, Mandarin,Portugusese and others languages. In Fig. 3 it is presented the Apple Siri lauched on an Iphone.

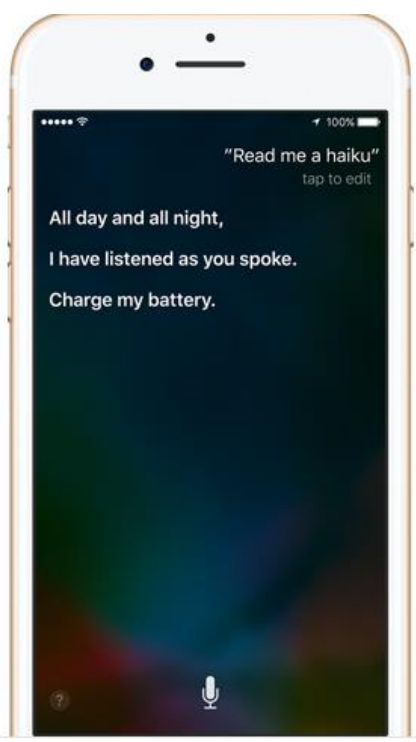

Fig. 3. - Siri launched on an iPhone 
The Microsoft Cortana is an Intelligent Personal Assistant that allows communication with the user through voice commands. It is capable of search online, dictate emails and solve mathematical equations. To answer the questions online, Microsoft Cortana uses the Bing Search Engine. The user can communicate with Microsoft Cortana in English, French, Spanish, Italian, Japanese and Mandarin This Intelligent Personal Assistant is integrated with Windows 10, Android, Xbox One and iOS platforms. In Fig. 4 it is presented the Microsoft Cortana launched in a Nokia smartphone.

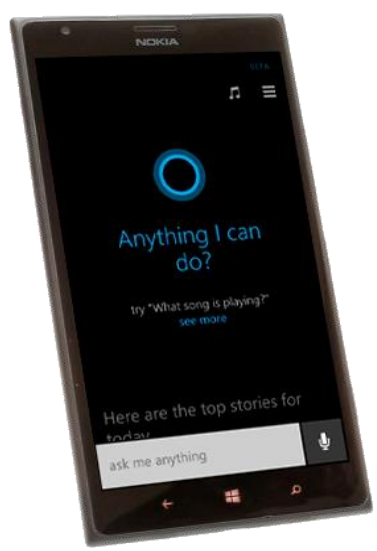

Fig. 4. - Microsoft Cortana launched on a Nokia smartphone

\section{Background}

The model, previously proposed [6], has two main tasks: (1) User identification and data acquisition related to user's personal information, context environment and state of mind assessment; (2) Proposal of a specific interaction activity, accordingly to the user's emotional state and context.

In the first task, the system will identify the user and assess his state of mind using image analysis. It will also gather contextual and personal data, e.g., special personal dates, physical location, or user's preferences.

In the second task, after the user identification and data acquisition phase, the retrieved data will guide the system on selecting and presenting some activities, accordingly to the user profile, current state of mind and context. For example, if the system detects that the user is sad, it can invite him to play one of his favorite games or listen to some of his usual music playlist.

\section{$3 \quad$ Methodology}

In this work we assess the usage of the current intelligent personals assistants according to the previously proposed model. We compare the features of each assistant with the 
relevant requirements of the proposal. The comparison should provide a good assessment, regarding how the intelligent personal assistant provides a set of basic features and requirements, correlated to the interaction of the user with five main features: (1) user identification; (2) state of mind assessment; (3) current context, (4) personal information acquisition; and (5) a set of activity proposals.

The user identification and state of mind evaluation will be made upon the features of image retrieval and analysis. The context and personal information, should include information such as: gender, age, personal preferences, time and date, physical location, etc.

The activity proposals, will be part of the possible interactions with the user. Some activities to be developed are: a) Basic greeting; b) Email management; c) Social Network management; d) Social and family events management; and e) Social games. In detail:

- The basic greeting activity is a simple greeting interaction, in which the system meets the user based upon the state of mind, time, and date.

- The email management activity should provide a personal email box to each user and inform the user about the current messages status. In specific dates, such as family anniversaries, the system should offer the user a chance to send a congratulations message.

- The social media management the system monitors the user group of family and friends, regarding their activity in the social networks, e.g., facebook, twitter, Instagram, etc., and informs the user about relevant updates.

- The social games activity should provide a set of social games, e.g., cards, trivia, etc., that the user can play in the system.

In Fig. 5, it is shown the system workflow of the proposed model in the previous work.

\section{System Model}

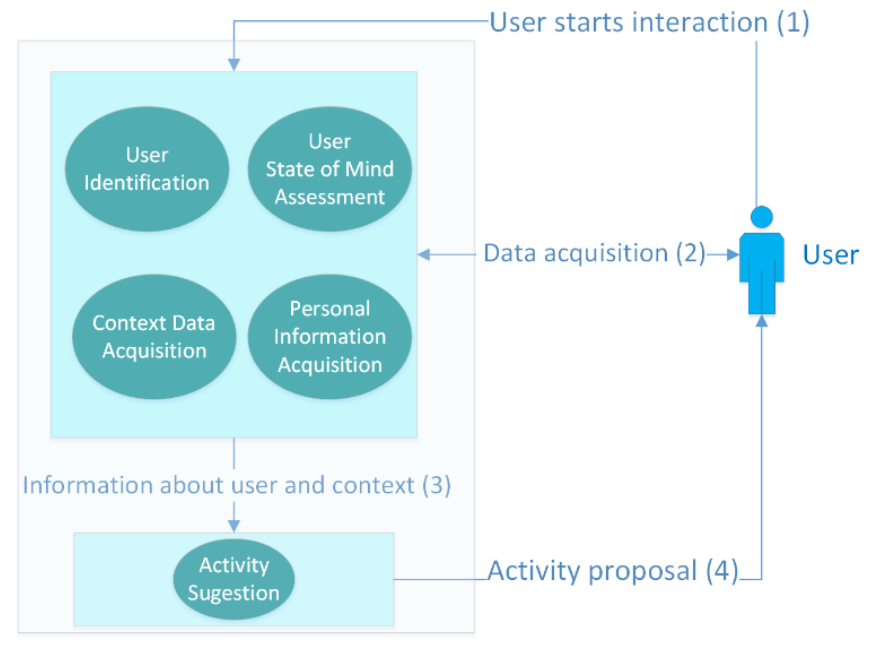

Fig. 5. - System workflow 
In the Fig. 5, it is presented the system's workflow, displaying the user interaction and the system response, acquiring the user's context and choosing an activity to interact with the user. All the communication is executed through natural language (voice) interactions.

\section{$4 \quad$ Assessment}

The following intelligent personal assistants systems were chosen for the assessment: (1) Google Assistant; (2) Amazon Alexa; (3) Apple Siri; and (4) Microsoft Cortana. These systems have Application Programming Interfaces (APIs) providing the functionalities for third party developers to customize some features, which may be used to implement the proposed model.

The assessment results are as follows:

1. The Google Assistant API [12], is divided in two categories: The first one is Conversation Actions, which help the developer to fulfil user requests by letting the developed system have a two-way dialog with users handling the interactions. The second category is Direct Actions that are currently in developing, it will be like Conversation Actions but it will make Google Assistant handle the user interaction, with the developer to be worried only about the handling of the developer's application. With Google Assistant, the user has many features like play news and music, check the weather and traffic, control smart devices like home temperature and lights, etc.

2. The Amazon Alexa API [13] permits developers to make their own application and connect them with users through command voices. Once integrated, the developed systems will have access to the built-in capabilities of Alexa (like music playback package tracking, movie listings, calendar management, and more) and third-party skills developed. The Amazon Alexa for users allows them to play news and music, check the weather and traffic, control smart devices like home temperature and lights, etc.

3. The Apple Siri API [14] handles all the user interaction, including the voice and natural language recognition, and works with the developers' application to get information and handle user requests. The developer can use 6 different categories of apps: audio and video calling apps; messaging apps; payment apps; apps that allow searching through photo libraries; workout apps; and ride booking apps. The Apple Siri for users allows them to play news and music, check the weather and traffic, control smart devices like home temperature and lights, etc.

4. The Microsoft Cortana API [15] is currently in developing expecting to be released in February 2017, which are a set of tools that allow app developers to integrate Cortana into their applications, interacting with voice commands. The Microsoft Cortana for users allows them to play news and music, check the weather and traffic, control smart devices like home temperature and lights, etc.

In Table 1, it is shown a comparison between the assessed intelligent personal assistants, related to the available features that might be used to implement a system for end users. 
Table 1. - Comparison of several features between the chosen Intelligent Personal Assistants

\begin{tabular}{|c|c|c|c|c|}
\hline & $\begin{array}{c}\text { Google } \\
\text { Assistant }\end{array}$ & $\begin{array}{c}\text { Amazon } \\
\text { Alexa } \\
\end{array}$ & $\begin{array}{l}\text { Apple } \\
\text { Siri }\end{array}$ & $\begin{array}{l}\text { Microsoft } \\
\text { Cortana } \\
\end{array}$ \\
\hline Create a shopping list & $\checkmark$ & $\checkmark$ & $x$ & $x$ \\
\hline Check Calendar & $\checkmark$ & $\checkmark$ & $\checkmark$ & $\checkmark$ \\
\hline Manage Calendar & $x$ & $\checkmark$ & $\checkmark$ & $\checkmark$ \\
\hline Check date and time & $\checkmark$ & $\checkmark$ & $\checkmark$ & $\checkmark$ \\
\hline Set an Alarm & $\checkmark$ & $\checkmark$ & $\checkmark$ & $\checkmark$ \\
\hline Check Weather & $\checkmark$ & $\checkmark$ & $\checkmark$ & $\checkmark$ \\
\hline Check Traffic & $\checkmark$ & $\checkmark$ & $\checkmark$ & $\checkmark$ \\
\hline Play news & $\checkmark$ & $\checkmark$ & $x$ & $x$ \\
\hline Play music & $\checkmark$ & $\checkmark$ & $\checkmark$ & $\checkmark$ \\
\hline Search Online & $\checkmark$ & $\checkmark$ & $\checkmark$ & $\checkmark$ \\
\hline Perform Calculations & $\checkmark$ & $\checkmark$ & $\checkmark$ & $\checkmark$ \\
\hline Call an Uber & $x$ & $\checkmark$ & $\checkmark$ & $x$ \\
\hline $\begin{array}{l}\text { Control home } \\
\text { temperature and lights }\end{array}$ & $\checkmark$ & $\checkmark$ & $\checkmark$ & $x$ \\
\hline Lock/Unlock Doors & $x$ & $x$ & $\checkmark$ & $x$ \\
\hline Play games & $\checkmark$ & $\checkmark$ & $\checkmark$ & $\checkmark$ \\
\hline Make a call & $\checkmark$ & $\checkmark$ & $\checkmark$ & $\checkmark$ \\
\hline Read books & $x$ & $\checkmark$ & $x$ & $x$ \\
\hline Shopping on amazon & $x$ & $\checkmark$ & $x$ & $x$ \\
\hline Order pizza & $x$ & $\checkmark$ & $\checkmark$ & $\checkmark$ \\
\hline Manage SMS & $\checkmark$ & $\checkmark$ & $\checkmark$ & $\checkmark$ \\
\hline Read emails & $x$ & $x$ & $\checkmark$ & $\checkmark$ \\
\hline Send emails & $x$ & $x$ & $\checkmark$ & $x$ \\
\hline $\begin{array}{l}\text { Send posts in } \\
\text { Facebook }\end{array}$ & $\checkmark$ & $\checkmark$ & $\checkmark$ & $x$ \\
\hline Send posts in Twitter & $\checkmark$ & $\checkmark$ & $\checkmark$ & $\checkmark$ \\
\hline $\begin{array}{c}\text { Receive Facebook } \\
\text { notifications }\end{array}$ & $x$ & $x$ & $x$ & $x$ \\
\hline $\begin{array}{c}\text { Receive } \\
\text { Twitter notifications }\end{array}$ & $x$ & $x$ & $x$ & $x$ \\
\hline
\end{tabular}

In Table 1, are shown several features that the Intelligent Personal Assistants are capable to provide, and can be used to implement users' interactions.

1. In Google Assistant, there are some important features like playing music, news, and the integration with Smart devices (control home lights and temperatures). But some features that are missing are the calendar and email management with voice commands interaction. 
2. In Amazon Alexa, it contains some important features like playing music, news, calendar management, book reader, and the integration with Smart devices (control home lights and temperatures). One feature that is missing is the email management with voice commands interaction.

3. In Apple Siri, it contains some important features like playing music, calendar and email management, and the integration with Smart devices (control home lights, temperature and door locks).

4. In Microsoft Cortana, it contains some important features like playing music, news, and calendar management. Some features that are missing are the email management and the integration with Smart devices (control home lights and temperatures) with voice commands interaction.

In all the Intelligent Personal Assistants, one important feature that was lacking is the social networks notifications.

From the features' set in the Methodology section, the Intelligent Personal Assistants should provide: (1) Basic greeting; (2) Email management; (3) Social Network management; (4) Social and family events management; and (5) Social games.

In the basic greeting activity, all available intelligent personal assistants have a strict greeting, though not allowing the users or other developers to customize. However, in Google Assistant and Amazon Alexa, both provide the possibility for the developer to create and publish their own application in their system, having the freedom to choose the interaction with the user.

In the email management activity, only Apple Siri and Microsoft Cortana can read emails. In specific dates, it is possible for the developer, in Amazon Alexa and Apple Siri, to send in birthday dates messages via Facebook, Twitter or SMS.

In the social network activity, the intelligent personal assistants are only capable of sending posts or update status.

In the playing games activity, all intelligent personal assistants are capable of playing games, e.g., trivia. A highlight goes to Microsoft Cortana that allows the user to play trivia games with other users.

\section{Conclusion}

In this paper, it was presented a comparison of several intelligent personal assistants, with the objective to evaluate how well these services would fulfil the proposed model, based on previous work. This services have many features in common, such as, playing music, search online, or playing games. Although it is important to know what are the features that each service provides, it is also important to understand the extent of how much an third party developer can use and customize these services to accomplish the proposed objectives. These objectives were the user's identification and some data acquisition like state of mind or context information to then propose some activities for voice interaction with the user, based upon the data gathered. These activities should be basic greeting, email management, social network events and social games.

The assessment presented in this paper, shows that it is possible in Amazon Alexa to fulfil the majority of these objectives: Basic greeting, to customize it would be better 
the Amazon Alexa or the Google Assistant; Email management, to read and send emails like in specific dates it should be used Amazon Alexa; Social Networks events, all services only allow the user's to post or update their status; Social Games, all services have social games like trivia but Microsoft Cortana allows the user's to play some games with other users.

In future work, it will be implemented a scenario, with some of these services and evaluated the effectiveness of the interaction with the users.

Acknowledgements. This work was supported by Project "NanoSTIMA: Macro-toNano Human Sensing: Towards Integrated Multimodal Health Monitoring and Analytics/NORTE-01-0145-FEDER-000016" financed by the North Portugal Regional Operational Programme (NORTE 2020), under the PORTUGAL 2020 Partnership Agreement, and through the European Regional Development Fund (ERDF).

\section{References}

1. Fernandes A (2001) Velhice, solidariedades familiares e política social: itinerário de pesquisa em torno do aumento da esperança de vida. Sociologia, Problemas e Práticas [online], n.36, pp.39-52.

2. Reis A, Reis C, Morgado L, Borges J, Tavares F, Gonçalves R, Cruz J (2016) Management of surgery waiting lists in the Portuguese public healthcare network: The information system for waiting list recovery programs. In Information Systems and Technologies (CISTI), 2016 11th Iberian Conference on (pp. 1-7). AISTI.

3. Palmer D, Newsom J, Rook K (2016) How does difficulty communicating affect the social relationships of older adults? An exploration using data from a national survey. Journal of Communication Disorders, 62:131-146.

4. Reis A, Lains J, Paredes H, Filipe V, Abrantes C, Ferreira F, Barroso, J. (2016). Developing a System for Post-Stroke Rehabilitation: An Exergames Approach. In International Conference on Universal Access in Human-Computer Interaction (pp. 403-413). Springer International Publishing.

5. Bassuk S, Glass A, Berkman F (1999) Social disengagement and incident cognitive decline in community-dwelling older persons. Annals of Internal Medicine, 131, 165-173. http://dx.doi.org/10.7326/0003-4819-131-3-199908030-00002

6. Reis A, Paredes H, Barroso I, Monteiro M, Rodrigues V, Khanal S, Barroso, J., (2016). Autonomous systems to support social activity of elderly people - A prospective approach to a system design. TISHW2016. International Conference on Technology and Innovation on Sports, Health and wellbeing. December 1-3, 2016 - UTAD, Vila Real, Portugal.

7. Wobcke W, Nguyen A, Ho H, Krzywicki A (2007) The Smart Personal Assistant: An Overview. In AAAI Spring Symposium: Interaction Challenges for Intelligent Assistants (pp. 135-136)

8. Google Assistant (2017) Google, accessed 20 January 2017, https://assistant.google.com

9. Amazon Alexa (2017) Amazon.com, accessed 20 January 2017, http://alexa.amazon.com/spa/index.html

10. Apple Siri (2017) Apple Inc., accessed 20 January 2017, http://www.apple.com/ios/siri/

11. Microsoft Cortana (2017), Microsoft, accessed 20 January 2017, https://www.microsoft.com/en/mobile/experiences/cortana/ 
12. Actions on Google (2017) Google, accessed 20 January 2017 , https://developers.google.com/actions/

13. Amazon Alexa Voice Service API (2017) Amazon.com, accessed 20 January 2017, https://developer.amazon.com/public/solutions/alexa/alexa-voice-service/content/avs-apioverview/

14. SiriKit - Apple Developer (2017) Apple Inc., accessed 20 January 2017, https://developer.apple.com/sirikit/

15. Microsoft Cortana Dev Center (2017), Microsoft, accessed 20 January 2017 https://developer.microsoft.com/en-us/cortana 